\title{
ALEXANDROV-FENCHEL TYPE INEQUALITIES IN THE SPHERE
}

\author{
MIN CHEN AND JUN SUN
}

\begin{abstract}
In this paper, we attempt to use two types of flows to study the relations between quermassintegrals $\mathcal{A}_{k}$ (see Definition 1.1), which correspond to the Alexandrov-Fenchel inequalities for closed convex $C^{2}$-hypersurfaces in $\mathbb{S}_{+}^{n+1}$.
\end{abstract}

\section{INTRODUCTION}

The Alexandrov-Fenchel inequalities [1, 2] for the quermassintegrals of convex domains in $\mathbb{R}^{n+1}$ are fundamental in classical geometry. In [11], Guan and Li extended these inequalities to star-shaped domains in $\mathbb{R}^{n+1}$. There have been extensive interests on studying the Alexandrov-Fenchel type inequalities for quermassintegral in space forms. Let $N^{n+1}(K)$ be the space form with constant sectional curvature $K=1,0$ or -1 . Under the Gaussian geodesic normal coordinates, the metric can be expressed as

$$
d s^{2}=d \rho^{2}+\phi^{2}(\rho) d z^{2},
$$

where $\phi(\rho)=\sin \rho, \rho \in\left[0, \frac{\pi}{2}\right)$ when $K=1 ; \phi(\rho)=\rho, \rho \in[0, \infty)$ when $K=0$; and $\phi(\rho)=\sinh \rho, \rho \in[0, \infty)$ when $K=-1$, and $d z^{2}$ is the induced standard metric on $\mathbb{S}^{n}$ in Euclidean space. We denote

$$
\Phi(\rho)=\int_{0}^{\rho} \phi(s) d s
$$

and consider the vector field $V=\phi(\rho) \frac{\partial}{\partial \rho}$. It is well known that $V$ is a conformal killing field. Let $M^{n} \subset \mathbb{N}^{n+1}(K)$ be a closed hypersurface and $\nu$ be the outward unit normal vector field. We call function $u=\langle V, \nu\rangle$ to be the generalized support function of the hypersurface.

Let $\kappa=\left\langle\kappa_{1}, \cdots, \kappa_{n}\right\rangle$ be the vector of $n$ principle curvatures of the hypersurface $M_{0}$ and we denote the $k$-th elementary symmetric function of $\kappa$ by $\sigma_{k}(\kappa)$. For

Key words and phrases. Alexandrov-Fenchel type inequalities, sphere, convexity.

MR(2010)Subject Classification 53C23, 35J60, 53C42.

The authors are supported by the National Nature Science Foudation of China No. 11721101 and National Key Research and Development Project No. SQ2020YFA070080. J. Sun is supported by NSFC 12071352. 
space form $\mathbb{N}^{n+1}$, there exists a notion of quermassintegrals, which can be expressed as a family of curvature integrals using Cauchy-Cronfton formulas (e.g., [17]).

Definition 1.1. Let $X: M \rightarrow \mathbb{N}^{n+1}(K)$ be a closed hypersurface embedded into $\mathbb{N}^{n+1}(K)$ and $\sigma_{k}$ be the $k$-th elementary symmetric function of the second fundamental form. Suppose $\Omega$ is the domain enclosed by $M^{n}$ in $\mathbb{N}^{n+1}(K)$. The $k$-th quermassintegal $\mathcal{A}_{k}$ is defined as follows:

$$
\begin{aligned}
& \mathcal{A}_{-1}(\Omega)=\operatorname{Vol}(\Omega), \\
& \mathcal{A}_{0}(\Omega)=\int_{M} d \mu_{g}, \\
& \mathcal{A}_{1}(\Omega)=\int_{M} \sigma_{1} d \mu_{g}+n K \operatorname{Vol}(\Omega), \\
& \mathcal{A}_{k}(\Omega)=\int_{M} \sigma_{k} d \mu_{g}+\frac{K(n-k+1)}{k-1} \mathcal{A}_{k-2}(\Omega),
\end{aligned}
$$

where $2 \leq k \leq n$.

In the hyperbolic space $\mathbb{H}^{n+1}$, there have been many interesting results. Brendle, Hung and Wang [5] used the inverse mean curvature flow to obtain Minkowski type inequality with weighted factor (the relation between $\int_{M} \sigma_{1} \phi^{\prime}$ and $\int_{\Omega} \phi^{\prime} d v o l$ ) for a compact mean convex hypersurface which is star-shaped with respect to the origin. In the case of $h$-convexity, full range of quermassintegral inequalities were obtained in [7, 20] using expanding and contracting types of flows. Very recently, the results in [20] for $h$-convex domains in $\mathbb{H}^{n+1}$ were reproved using flow (1.1) directly in [14 by establishing that $h$-convexity is preserved along flow (1.1). The sharp relation between $\mathcal{A}_{2}$ and $\mathcal{A}_{0}$ was previously proved in [15] by a different method. Andrews, Chen and Wei [3] replaced the $h$-convexity assumption with the weaker assumption of positive sectional curvature to obtain the relation between $\mathcal{A}_{k}$ and $\mathcal{A}_{-1}$ for $0 \leq k \leq n-1$. In the general case of $k$-convexity, Brendle, Guan and Li [4] considered the following parabolic evolution equation of a smooth one parameter family of embedded hypersurfaces $X(\cdot, t) \subset \mathbb{N}^{n+1}(K)$,

$$
X_{t}=\left(\frac{\phi^{\prime}(\rho)}{F}-\frac{u}{c_{n, k}}\right) \nu,
$$

where $X$ is the position vector and $F:=\frac{\sigma_{k+1}(\kappa)}{\sigma_{k}(\kappa)}$. The motivation to study BrendleGuan-Li' flow is the Alexandrov-Fenchel type inequalities for quermassintegrals in space forms. If one establishes long time existence and convergence of the flow (1.1), the sharp Alexandrov-Fenchel inequalities for $k$-star-shaped domains in $\mathbb{N}^{n+1}(K)$ would follow. In the case $K=-1$, the main problem is the preservation of star-shapedness along the flow. As a consequence, Brendle, Guan and Li [4] established sharp inequalities between $\mathcal{A}_{n-1}$ and $\mathcal{A}_{k}$ for convex domains in $\mathbb{H}^{n+1}$, $\forall k \leq n-1$. For general $0 \leq k \leq l \leq n-1$ in $\mathbb{H}^{n+1}$, with an extra initial gradient 
bound Condition $\left(\max _{x \in \mathbb{S}^{n}}|\nabla \ln (\cosh \rho)|^{2}(x, 0) \leq 12+3 \min _{t=0} \sinh ^{2} \rho\right.$ ), Brendle, Guan and Li [4] can obtain the sharp inequalities of the relations between $\mathcal{A}_{k}$ and $\mathcal{A}_{l}$.

In $\mathbb{S}^{n+1}$, Girão and Pinheiro [9] used the inverse mean curvature flow to prove the Minkowski type inequality. Brendle, Guan and Li [4] established sharp inequalities between $\mathcal{A}_{n-1}$ and $\mathcal{A}_{k}$ for convex domains in $\mathbb{S}^{n+1}, \forall k \leq n-1$. Wei and Xiong proved the following optimal inequalities for convex hypersurfaces in sphere.

Theorem 1.2. (see Theorem 1.3 in [21]) Let $\Sigma^{n}$ be a closed and strictly convex hypersurface in $\mathbb{S}^{n+1}$. Then we have the following optimal inequalities $\left(k \leq \frac{n}{2}\right)$

$$
\int_{\Sigma} L_{k} d \mu \geq C_{n}^{2 k}(2 k) ! \omega_{n}^{\frac{2 n}{k}}|\Sigma|^{\frac{n-2 k}{n}} .
$$

Equality holds if and only if $\Sigma$ is a geodesic sphere.

Here, the Gauss-Bonnet curvature is

$$
L_{k}=C_{n}^{2 k}(2 k) ! \sum_{i=0}^{k} \frac{C_{k}^{i}}{C_{n}^{2 k-2 i}}(-1)^{i} \sigma_{2 k-2 i} .
$$

Makowski and Scheuer proved the following Alexandrov-Fenchel type inequalities in the spheres. Namely

Theorem 1.3. (see Theorem 7.6 in [16] ) Let $M \subset \mathbb{S}^{n+1}$ be an embedded, closed connected and convex $C^{2}$-hypersurface of the sphere. Let $k \in \mathbb{N}_{+}$with $2 k+1 \leq n$ and let $\hat{M}$ be convex body enclosed by $M$. Then we have the inequality

$$
W_{2 k+1}(\hat{M}) \geq \frac{\omega_{n}}{n+1} \sum_{i=0}^{k}(-1)^{i} \frac{n-2 k}{n-2 k+2 i}\left(\begin{array}{c}
k \\
i
\end{array}\right)\left(\frac{n+1}{\omega_{n}} W_{1}(\hat{M})\right)^{\frac{n-2 k+2 i}{n}},
$$

and equality holds if and only if $M$ is a geodesic sphere.

By Proposition 7 in [18], we have

$$
W_{k+1}=\frac{1}{(n+1)\left(\begin{array}{l}
n \\
k
\end{array}\right)} \mathcal{A}_{k} .
$$

In view of (1.2), Theorem 1.3 implies the relation between $A_{2 k}$ and $A_{0}$ in $\mathbb{S}^{n+1}$ (Ge, Wang and Wu also proved a similar type of inequalities in $\mathbb{H}^{n+1}$ in Theorem 1.3 in [7] before). The optimal inequalities between two quermasssintegrals $\mathcal{A}_{k}$ and $\mathcal{A}_{l}$ (for general $0 \leq k \leq l \leq n-1$ ) in $\mathbb{S}^{n+1}$ are still open. We will derive the relation between two adjacent quermassintegrals for general even number (i.e., $A_{2 k}$ and $\left.A_{2 k-2}\right)$. We will also derive the relation between two adjacent quermassintegrals for general odd number (i.e., $A_{2 k+1}$ and $A_{2 k-1}$ ) as well.

Assume that $s_{k}=\mathcal{A}_{k}\left(B_{\frac{\pi}{2}}(o)\right)$, we now state the main result of this paper: 
Theorem 1.4. Let $M$ be a closed convex $C^{2}$-hypersurface in $\mathbb{S}_{+}^{n+1}$, then the following inequality holds,

$$
\mathcal{A}_{k}(\Omega) \geq \xi_{k, k-2}\left(\mathcal{A}_{k-2}(\Omega)\right) \quad \text { for any } \quad 1 \leq k \leq n-1,
$$

where $\xi_{k, k-2}$ is the unique positive function defined on $\left(0, s_{k-2}\right)$ such that the equality holds if and only if $M$ is a geodesic sphere.

We will prove the relations between $\mathcal{A}_{k}$ and $\mathcal{A}_{k-2}$ by induction. It is already known that hypersurfaces converge smoothly to the equator along the Gerhadt's flow (see flow (4.1)). In Section 4, under the assumption that $\mathcal{A}_{k-2}(\Omega) \geq$ $\xi_{k-2, k-4}\left(\mathcal{A}_{k-4}(\Omega)\right)$ (the equality holds if and only if $M$ is a geodesic sphere), we could apply Gerhardt's flow to prove that $\mathcal{A}_{k}(\Omega) \geq \xi_{k, k-2}\left(\mathcal{A}_{k-2}(\Omega)\right)$ (the equality holds if and only if $M$ is a geodesic sphere). We can also use Gerhardt's flow to derive the relation between $\mathcal{A}_{2}$ and $\mathcal{A}_{0}$ as well. However, we are unable to use Gerhardt's flow to obtain the relation between $\mathcal{A}_{1}$ and $\mathcal{A}_{-1}$ as we are failed to get enough information about $\xi_{1,-1}$ as we describe in (4.15) (see also (4.9)) by the definition of $\xi_{k, k-2}$. To derive the relation of $\mathcal{A}_{1}$ and $\mathcal{A}_{-1}$, we will use Chen-Guan-Li-Scheuer's flow (see flow (3.1)) in Section 3. A nice feature of this type of flow is that we do not need to know specific information about $\xi_{1,-1}$ and we just need to obtain the monotonicity property of $\mathcal{A}_{1}$ and $\mathcal{A}_{-1}$ along the flow to acquire the relation between $\mathcal{A}_{1}$ and $\mathcal{A}_{-1}$. More generally, we can prove the relation between $\mathcal{A}_{m}$ and $\mathcal{A}_{-1}$ for $0 \leq m \leq n-1$. Except in the case of $k=0$ (i.e., flow (3.2) ) where $C^{2}$ estimates follow directly from the theory of quasi-linear PDE, $C^{2}$ estimates for solutions of flow (3.1) is still an open question. As a result, combining applying Gerhardt's flow with Chen-Guan-Li-Scheuer's flow together, we could establish a full range of Alexandrov-Fenchel inequalities described in Theorem 1.4.

The subsequent sections of this paper are organized as follows: in Section 2, we will recall some general facts on the elementary symmetric functions and the quermassintegrals; in Section 3, we will use Chen-Guan-Li-Scheuer's flow to prove the the relation between $\mathcal{A}_{m}$ and $\mathcal{A}_{-1}$; in Section 4, we will use Gerhardt's flow to finish the proof of the main theorem.

\section{Setting AND General FaCts}

Let us present some basic facts which will be used later in this paper.

Definition 2.1. (10]) For $1 \leq k \leq n$, let $\Gamma_{k}$ be a cone in $\mathbb{R}^{n}$ determined by

$$
\Gamma_{k}=\left\{\lambda \in \mathbb{R}^{n}: \sigma_{1}>0, \cdots, \sigma_{k}>0\right\} .
$$

An $n \times n$ symmetric matrix $W$ is called belonging to $\Gamma_{k}$ if $\lambda(W) \in \Gamma_{k}$.

Then we will introduce Newton-Maclaurin inequalities and Minkowski identity. 
Lemma 2.2. ([10]) For $W \in \Gamma_{k}$,

$$
(n-k+1)(k+1) \sigma_{k-1}(W) \sigma_{k+1}(W) \leq k(n-k) \sigma_{k}^{2}(W),
$$

and

$$
\sigma_{k+1}(W) \leq c_{n, k} \sigma_{k}^{\frac{k+1}{k}}(W)
$$

where $c_{n, k}=\frac{\sigma_{k}^{\frac{k+1}{k}}}{\sigma_{k+1}}(I)$. The equality holds if and only if $W=c I$ for some $c>0$.

Proposition 2.3. (4] Let $M$ be a closed hypersurfaces in $\mathbb{N}^{n+1}(K)$. Then, for $k=0,1, \cdots, n-1$,

$$
(k+1) \int_{M} \sigma_{k+1} u=(n-k) \int_{M} \phi^{\prime}(\rho) \sigma_{k}(\kappa) .
$$

We will use the convention that $\sigma_{0} \equiv 1$.

We define

$$
\Phi(\rho)=\int_{0}^{\rho} \phi(r) d r
$$

Then $\Phi(\rho)$ is $\frac{\rho^{2}}{2}, \cosh \rho-1,1-\cos \rho$ for $K=0,-1$ and 1 , respectively. The following lemma holds for general warped product manifolds.

Lemma 2.4. (12]) Let $M^{n} \subset N^{n+1}$ be a closed hypersurface with induced metric g. Let $\Phi$ be defined as in (2.1) and $V=\phi(\rho) \frac{\partial}{\partial \rho}$. Then $\left.\Phi\right|_{M}$ satisfies,

$$
\nabla_{i} \nabla_{j} \Phi=\phi^{\prime}(\rho) g_{i j}-h_{i j}\langle V, \nu\rangle,
$$

where $\nabla$ is the covariant derivative with respect to $g, \nu$ is the outward unit normal, and $h_{i j}$ is the second fundamental form of the hypersurface.

We also recall the gradient and hessian of the support function $u:=\langle V, \nu\rangle$ under the induced metric $g$ on $M$.

Lemma 2.5. ([12]) The support function u satisfies

$$
\begin{aligned}
& \nabla_{i} u=h_{i l} \nabla_{l} \Phi \\
& \nabla_{i} \nabla_{j} u=\nabla_{l} h_{i j} \nabla_{l} \Phi+\phi^{\prime} h_{i j}-u\left(h^{2}\right)_{i j},
\end{aligned}
$$

where $\left(h^{2}\right)_{i j}:=g^{k l} h_{i k} h_{j l}$.

Next we will present the evolution equations of $\sigma_{l}$ and quermassintegrals. Let $M(t)$ be a smooth family of closed hyersurfaces in $\mathbb{N}^{n+1}(K)$. Let $X(\cdot, t)$ denote a point on $M(t)$. We will consider the flow

$$
X_{t}=f \nu .
$$

Along this flow, we have 
Proposition 2.6. (44) Under the flow (2.2) for the hypersurface in a Riemannian manifold, suppose $\Omega$ is the domain enclosed by the closed hypersurface, we have the following evolution equations.

$$
\begin{aligned}
\partial_{t} g_{i j} & =2 f h_{i j} \\
\partial_{t} h_{i j} & =-\nabla_{i} \nabla_{j} f+f\left(h^{2}\right)_{i j}-f R_{\nu i j \nu} \\
\partial_{t} h_{i}^{j} & =-g^{j k} \nabla_{k} \nabla_{j} f-g^{j k} f\left(h^{2}\right)_{k i}-f g^{j k} R_{\nu i k \nu} \\
\partial_{t} \sigma_{k} & =\frac{\partial \sigma_{k}}{\partial h_{i}^{j}} \partial_{t} h_{i}^{j}
\end{aligned}
$$

Moreover, if $N$ has constant sectional curvature $K$, then for $l \geq 0$, we have

$$
\partial_{t} \int_{M} \sigma_{l}=\int_{M} f\left[(l+1) \sigma_{l+1}-(n-l+1) K \sigma_{l-1}\right] d \mu_{g},
$$

and

$$
\partial_{t} \operatorname{Vol}(\Omega)=\int_{M} f d \mu_{g}
$$

Using Proposition 2.6, we have the following proposition, which motivates the definition of the quermassintegrals:

Proposition 2.7. (4]) In $\mathbb{N}^{n+1}(K)$, along the flow (2.2) for $0 \leq l<n-1$, we have

$$
\partial_{t} \mathcal{A}_{l}=(l+1) \int_{M} f \sigma_{l+1} .
$$

Let $B_{\rho}(o) \subset \mathbb{N}^{n+1}(K)$ be the geodesic ball of radius $\rho$ centered at the origin $o$. Then we have

$$
\frac{d}{d \rho}\left(\mathcal{A}_{k}\left(B_{\rho}(o)\right)\right)=(k+1) \int_{\partial B_{\rho}(o)} \sigma_{k+1}>0,
$$

for any $k=-1,0,1,2, \cdots, n-1$. If we view $\mathcal{A}_{k}\left(B_{\rho}(o)\right)$ as a function of $\rho$, then the inverse function can be denoted as

$$
\rho=\eta_{k}\left(\mathcal{A}_{k}\left(B_{\rho}(o)\right)\right),
$$

where $\eta_{k}:\left(0, s_{k}\right) \rightarrow(0, \pi / 2)$ is a strictly increasing function for any fixed $k$. Brendle, Guan and Li [4] want to compare the relation between $\mathcal{A}_{k}$ and $\mathcal{A}_{l}$ for given $k>l$ and balls $B_{\rho}(o), \rho>0$ in $\mathbb{N}^{n+1}(K)$, which are the optimal solutions of this isoperimetric problem. Let $\xi_{k, l}$ be the unique positive strictly increasing function defined on $\left(0, s_{l}\right)$ such that

$$
\mathcal{A}_{k}\left(B_{\rho}(o)\right)=\xi_{k, l}\left(\mathcal{A}_{l}\left(B_{\rho}(o)\right)\right) .
$$

In general, for a bounded domain $\Omega \subset \mathbb{N}^{n+1}(K)$, they want to establish

$$
\mathcal{A}_{k}(\Omega) \geq \xi_{k, l}\left(\mathcal{A}_{l}(\Omega)\right)
$$


In this following part of this paper, we will prove the relation between $\mathcal{A}_{k}$ and $\mathcal{A}_{k-2}$. More precisely, we will show that

$$
\mathcal{A}_{k}(\Omega) \geq \xi_{k, k-2}\left(\mathcal{A}_{k-2}(\Omega)\right) \text {. }
$$

\section{Chen-Guan-Li-Scheuer's flow and applications}

In this section, we will establish the relation between $\mathcal{A}_{1}(\Omega)$ and $\mathcal{A}_{-1}(\Omega)$. Actually, we will provide the relationship between $\mathcal{A}_{m}$ and $\mathcal{A}_{-1}$ for $1 \leq m \leq n-1$. In [6], Chen, Guan, Li and Scheuer introduced the following flow

$$
X_{t}=\left(c_{n, k} \phi^{\prime}-\frac{\sigma_{k+1}}{\sigma_{k}} u\right) \nu,
$$

Similar to the Brendle-Guan-Li's flow, monotonicity property for quermassintegrals holds as long as the flow (3.1) exists. It is claimed in [13] and proved in [6] that flow (3.1) preserves convexity, and one has $C^{1}$-estimates for solutions, and upper and lower bounds for $F=\frac{\sigma_{k+1}}{\sigma_{k}}$ along the flow (3.1). For completeness, we will include a proof of convexity preserving which we will use later here.

3.1. Convexity preserving. In this subsection, we will prove that if $M$ is strictly convex, then along the normalized flow (3.1), the principle curvatures of $M_{t}$ remain strictly positive. We will need the following algebraic lemma.

Lemma 3.1. ([19]) Let $F=\frac{\sigma_{k+1}}{\sigma_{k}}\left(h_{i j}\right)$, and $\left\{\tilde{h}^{i j}\right\}$ be the inverse matrix of $h_{i j}$, then

$$
\left(F^{i j, r s}+2 F^{i r} \tilde{h}^{j s}\right) \eta_{i j} \eta_{r s} \geq 2 F^{-1}\left(F^{i j} \eta_{i j}\right)^{2},
$$

for any real symmetric $n \times n$ matrix $\left(\eta_{i j}\right)$. Here, $F^{i j}=\frac{\partial F}{\partial h_{i j}}$ and $F^{i j, r s}=\frac{\partial^{2} F}{\partial h_{i j} \partial h_{r s}}$.

Lemma 3.2. Let $X(\cdot, t)$ be a smooth, closed and strictly convex solution to the normalized flow (3.1) for $t \in[0, T)$, which encloses the origin. There is a positive constant $C$ depending only on $M$, the upper and lower bounds of $\rho\left(C^{0}\right.$ estimate), the gradient estimate $\rho\left(C^{1}\right.$ estimate), and the upper bound of $F$ such that the principal curvatures of $X(\cdot, t)$ are bounded from below

$$
\kappa_{i}(\cdot, t) \geq \frac{1}{C}, \quad \forall t \in[0, T) \quad \text { and } i=1, \cdots, n .
$$

Proof. From Proposition 2.6 and using the fact that $\nabla_{i} \nabla_{j} \phi^{\prime}=-K \nabla_{i} \nabla_{j} \Phi$, we compute

$$
\begin{aligned}
\partial_{t} h_{i j} & =-\nabla_{i} \nabla_{j}\left(c_{n, k} \phi^{\prime}-u F\right)+\left(c_{n, k} \phi^{\prime}-u F\right)\left(h^{2}\right)_{i j}-K\left(c_{n, k} \phi^{\prime}-u F\right) \delta_{j}^{i} \\
& =-\left(c_{n, k} \nabla_{i} \nabla_{j} \phi^{\prime}-\nabla_{i} \nabla_{j} u F-\nabla_{i} u \nabla_{j} F-\nabla_{i} F \nabla_{j} u-u \nabla_{i} \nabla_{j} F\right) \\
& +\left(c_{n, k} \phi^{\prime}-u F\right)\left(h^{2}\right)_{i j}-K\left(c_{n, k} \phi^{\prime}-u F\right) \delta_{j}^{i} \\
& =K c_{n, k} \nabla_{i} \nabla_{j} \Phi+\nabla_{i} \nabla_{j} u F+\nabla_{i} u \nabla_{j} F+\nabla_{i} F \nabla_{j} u+u \nabla_{i} \nabla_{j} F \\
& -\left(c_{n, k} \phi^{\prime}-u F\right)\left(h^{2}\right)_{i j}-K\left(c_{n, k} \phi^{\prime}-u F\right) \delta_{j}^{i}
\end{aligned}
$$




$$
\begin{aligned}
& =K c_{n, k}\left(\phi^{\prime} g_{i j}-u h_{i j}\right)+\left(\nabla h_{i j} \nabla \Phi+\phi^{\prime} h_{i j}-u\left(h^{2}\right)_{i j}\right) F+h_{i l} \nabla_{l} \Phi \nabla_{j} F+h_{j l} \nabla_{l} \Phi \nabla_{i} F \\
& +u\left(F^{k l} \nabla_{k} \nabla_{l} h_{i j}+\left(F^{k l}\left(h^{2}\right)_{k l}-K F^{k k}\right) h_{i j}-F\left(\left(h^{2}\right)_{i j}-K g_{i j}\right)+F^{k l, p q} \nabla_{i} h_{p q} \nabla_{j} h_{k l}\right) \\
& +\left(c_{n, k} \phi^{\prime}-u F\right)\left(h^{2}\right)_{i j}-K\left(c_{n, k} \phi^{\prime}-u F\right) \delta_{j}^{i} .
\end{aligned}
$$

Thus

$$
\begin{aligned}
\partial_{t} h_{i j} & =u F^{k l} \nabla_{k} \nabla_{l} h_{i j}+u F^{k l, p q} \nabla_{i} h_{p q} \nabla_{j} h_{k l}+\nabla h_{i j} \nabla \Phi F+h_{i l} \nabla_{l} \Phi \nabla_{j} F+h_{j l} \nabla_{l} \Phi \nabla_{i} F \\
& +\left(c_{n, k} \phi^{\prime}-3 u F\right)\left(h^{2}\right)_{i j}+\left(\left(F^{k l}\left(h^{2}\right)_{k l}-K F^{k k}\right) u-c_{n, k} K u+\phi^{\prime} F\right) h_{i j}+2 K u F \delta_{j}^{i} .
\end{aligned}
$$

Assume $\left(\tilde{h}^{i j}\right)$ to be the inverse matrix of $\left(h_{i j}\right)$. The principle radii of curvature of $M_{t}$ are the eigenvalues of $\left\{\tilde{h}^{i k} g_{k j}\right\}$. To derive a positive lower bound of principle curvatures, it suffices to prove that the eigenvalues of $\left(\tilde{h}^{i k} g_{k j}\right)$ are bounded from above. For this, we consider the following quantity

$$
W(x, t)=\log \Lambda(x, t)-\log u(x, t),
$$

where

$$
\Lambda(x, t)=\max \left\{\tilde{h}^{i j}(x, t) \xi_{i} \xi_{j}: g^{i j}(x, t) \xi_{i} \xi_{j}=1\right\} .
$$

Assume $W$ attains its maximum on $\mathbb{S}^{n} \times\left[0, T^{\prime}\right]$ at $\left(x_{0}, t_{0}\right)$ for any fixed $T^{\prime}<T$. We choose a local orthonormal frame $e_{1}, e_{2}, \cdots, e_{n}$ on $M_{t}$ such that $\left(h_{i j}\right)$ is diagonal at $F\left(x_{0}, t_{0}\right)$. By a rotation, we may also suppose that $\Lambda\left(x_{0}, t_{0}\right)=\tilde{h}^{i j}(x, t) \xi_{i} \xi_{j}$ with $\xi=(1,0, \cdots, 0)$. Let

$$
\omega(x, t)=\log \lambda(x, t)-\log u(x, t),
$$

where $\lambda(x, t)=\tilde{h}^{11} / g^{11}$. Then $\max _{\mathbb{S}^{n} \times\left[0, T^{\prime}\right]} W=\max _{\mathbb{S}^{n} \times\left[0, T^{\prime}\right]} \omega$ and so $\omega$ achieves its maximum at $\left(x_{0}, t_{0}\right)$. In the following we prove an upper bound for $\omega$.

$$
\begin{aligned}
\partial_{t} \lambda= & -\left(\tilde{h}^{11}\right)^{2} \partial_{t} h_{11}+\tilde{h}^{11} \partial_{t} g_{11} \\
= & -\left(\tilde{h}^{11}\right)^{2}\left(u F^{k l} \nabla_{k} \nabla_{l} h_{11}+u F^{k l, p q} \nabla_{1} h_{p q} \nabla_{1} h_{k l}+\nabla h_{11} \nabla \Phi F+h_{1 l} \nabla_{l} \Phi \nabla_{1} F\right. \\
& +h_{1 l} \nabla_{l} \Phi \nabla_{1} F+\left(c_{n, k} \phi^{\prime}-3 u F\right)\left(h^{2}\right)_{11} \\
& \left.+\left(\left(F^{k l}\left(h^{2}\right)_{k l}-K F^{k k}\right) u-c_{n, k} K u+\phi^{\prime} F\right) h_{11}+2 K u F\right)+2\left(c_{n, k} \phi^{\prime}-u F\right) \\
= & -\left(\tilde{h}^{11}\right)^{2}\left(u F^{k l} \nabla_{k} \nabla_{l} h_{11}+u F^{k l, p q} \nabla_{1} h_{p q} \nabla_{1} h_{k l}\right)+\nabla \lambda \nabla \Phi F-2 \tilde{h}^{11} \nabla_{1} \Phi \nabla_{1} F \\
& -\left(c_{n, k} \phi^{\prime}-3 u F\right)-\tilde{h}^{11}\left(\left(F^{k l}\left(h^{2}\right)_{k l}-K F^{k k}\right) u-c_{n, k} K u+\phi^{\prime} F\right) \\
& -2 K u F\left(\tilde{h}^{11}\right)^{2}+2\left(c_{n, k} \phi^{\prime}-u F\right) \\
= & u F^{k l} \nabla_{k} \nabla_{l} \lambda-2 u F^{k l}\left(\tilde{h}^{11}\right)^{2} \tilde{h}^{p q} \nabla_{1} h_{k p} \nabla_{1} h_{l q}-u\left(\tilde{h}^{11}\right)^{2} F^{k l, p q} \nabla_{1} h_{p q} \nabla_{1} h_{k l} \\
& +\nabla \lambda \nabla \Phi F-2 \tilde{h}^{11} \nabla_{1} \Phi \nabla_{1} F+\left(c_{n, k} \phi^{\prime}+u F\right) \\
& -\tilde{h}^{11}\left(\left(F^{k l}\left(h^{2}\right)_{k l}-K F^{k k}\right) u-c_{n, k} K u+\phi^{\prime} F\right)-2 K u F\left(\tilde{h}^{11}\right)^{2},
\end{aligned}
$$


where we used the fact that

$$
\begin{aligned}
\nabla_{i} \lambda & =-\left(\tilde{h}^{11}\right)^{2} h_{11, i} \\
\nabla_{i} \nabla_{j} \lambda & =-\left(\tilde{h}^{11}\right)^{2} \nabla_{i} \nabla_{j} h_{11}+2 \tilde{h}^{p q} \nabla_{1} h_{i p} \nabla_{1} h_{j q} .
\end{aligned}
$$

By Lemma 3.1, we have

$$
\begin{aligned}
\partial_{t} \lambda & \leq u F^{k l} \nabla_{k} \nabla_{l} \lambda-2 u F^{-1}\left(\tilde{h}^{11}\right)^{2}\left(\nabla_{1} F\right)^{2}+\nabla \lambda \nabla \Phi F-2 \tilde{h}^{11} \nabla_{1} \Phi \nabla_{1} F \\
& +\left(c_{n, k} \phi^{\prime}+u F\right)-\tilde{h}^{11}\left(\left(F^{k l}\left(h^{2}\right)_{k l}-K F^{k k}\right) u-c_{n, k} K u+\phi^{\prime} F\right) \\
& -2 K u F\left(\tilde{h}^{11}\right)^{2} .
\end{aligned}
$$

On the other hand,

$$
\begin{aligned}
& \partial_{t} u-u F^{k l} u_{k l} \\
= & f \phi^{\prime}-\nabla \Phi \nabla f-u F^{k l} u_{k l} \\
= & \left(c_{n, k} \phi^{\prime}-u F\right) \phi^{\prime}-\nabla \Phi \nabla\left(c_{n, k} \phi^{\prime}-u F\right)-u F^{k l}\left(\nabla h_{k l} \nabla \Phi+\phi^{\prime} h_{k l}-u\left(h^{2}\right)_{k l}\right) \\
= & c_{n, k}\left(\phi^{\prime}\right)^{2}+K c_{n, k}\left|\nabla \phi^{\prime}\right|^{2}+\nabla \Phi \nabla u F-2 u \phi^{\prime} F+u^{2} F^{k l}\left(h^{2}\right)_{k l} .
\end{aligned}
$$

Note that, at $\left(x_{0}, t_{0}\right)$,

$$
\nabla_{i} \log \lambda=\nabla_{i} \log u, \quad \forall i=1, \cdots, n,
$$

and

Then we have

$$
\nabla_{i} \nabla_{j}(\log \lambda-\log u) \leq 0 .
$$

$$
\begin{aligned}
\frac{1}{\lambda u}\left(u^{2} F^{k l} \nabla_{k} \nabla_{l} \lambda-u \lambda F^{k l} \nabla_{k} \nabla_{l} u\right) & =u F^{k l} \nabla_{k} \nabla_{l}(\log \lambda-\log u), \\
\frac{1}{\lambda u}(u F \nabla \lambda \nabla \Phi-\lambda F \nabla \Phi \nabla u) & =0 .
\end{aligned}
$$

Thus

$$
\begin{aligned}
0 & \leq \partial_{t}(\log \lambda-\log u)=\frac{\lambda_{t}}{\lambda}-\frac{u_{t}}{u} \\
& \leq \frac{1}{\lambda u}\left(u^{2} F^{k l} \nabla_{k} \nabla_{l} \lambda-2 u^{2} F^{-1}\left(\tilde{h}^{11}\right)^{2}\left(\nabla_{1} F\right)^{2}+u F \nabla \lambda \nabla \Phi-2 u \tilde{h}^{11} \nabla_{1} \Phi \nabla_{1} F\right. \\
& +u\left(c_{n, k} \phi^{\prime}+u F\right)-\tilde{h}^{11}\left(\left(F^{k l}\left(h^{2}\right)_{k l}-K F^{k k}\right) u^{2}-c_{n, k} K u^{2}+\phi^{\prime} u F\right) \\
& -2 K u^{2} F\left(\tilde{h}^{11}\right)^{2}-\left(\lambda c_{n, k}\left(\phi^{\prime}\right)^{2}+\lambda K c_{n, k}\left|\nabla \phi^{\prime}\right|^{2}+\nabla \Phi \nabla u \lambda F-2 u \lambda \phi^{\prime} F\right. \\
& \left.\left.\left.+\lambda u^{2} F^{k l}\left(h^{2}\right)^{k l}\right)+u \lambda F^{k l} \nabla_{k} \nabla_{l} u\right)\right) \\
& =u F^{k l} \nabla_{k} \nabla_{l}(\log \lambda-\log u)+\frac{1}{\lambda u}\left(\left(-2 u^{2} F^{-1}\left(\tilde{h}^{11}\right)^{2}\left(\nabla_{1} F\right)^{2}-2 u \tilde{h}^{11} \nabla_{1} \Phi \nabla_{1} F\right)\right. \\
& -2 K u^{2}\left(\tilde{h}^{11}\right)^{2}-\tilde{h}^{11}\left(\left(2 F^{k l}\left(h^{2}\right)_{k l}-K F^{k k}\right) u^{2}-c_{n, k} K u^{2}-\phi^{\prime} u F-c_{n, k}\left(\phi^{\prime}\right)^{2}\right. \\
& \left.\left.+K c_{n, k}\left|\nabla \phi^{\prime}\right|^{2}\right)+u\left(c_{n, k} \phi^{\prime}+u F\right)\right)
\end{aligned}
$$




$$
\begin{aligned}
& =u F^{k l} \nabla_{k} \nabla_{l}(\log \lambda-\log u)+\frac{1}{\lambda u}\left(\frac{1}{2} F\left(\nabla_{1} \Phi\right)^{2}-2 K u^{2}\left(\tilde{h}^{11}\right)^{2}\right. \\
& -\tilde{h}^{11}\left(\left(2 F^{k l}\left(h^{2}\right)_{k l}-K F^{k k}\right) u^{2}-c_{n, k} K u^{2}-\phi^{\prime} u F-c_{n, k}\left(\phi^{\prime}\right)^{2}+K c_{n, k}\left|\nabla \phi^{\prime}\right|^{2}\right) \\
& \left.+u\left(c_{n, k} \phi^{\prime}+u F\right)\right) .
\end{aligned}
$$

Now we assume $K=1$. It implies that

$$
\begin{aligned}
& -2 u^{2}\left(\tilde{h}^{11}\right)^{2}+\tilde{h}^{11}\left(F^{k k} u^{2}+c_{n, k}\left(u^{2}+\left(\phi^{\prime}\right)^{2}-\left|\nabla \phi^{\prime}\right|^{2}\right)+u \phi^{\prime} F\right) \\
& +u\left(c_{n, k} \phi^{\prime}+u F\right)+\frac{1}{2} F\left(\nabla_{1} \Phi\right)^{2} \geq 0 .
\end{aligned}
$$

Then we have

$$
\left(\tilde{h}^{11}\right)^{2}-c_{3} \tilde{h}^{11}-c_{4} \leq 0
$$

where the positive constants $c_{3}, c_{4}$ depend on the upper and lower bound of $\rho$, the lower bound of $u$ and the upper bound of $F$. Since $u=\frac{\phi^{2}}{\sqrt{\phi^{2}+|\nabla \rho|^{2}}}$ ( see equation (4.1) in [12]), we can conclude that the lower bound of $u$ depends on the gradient estimate of $\rho$ ( $C^{1}$ estimate).

The upper bound for $\tilde{h}^{11}$ implies an upper bound for $\omega$ since $u$ is bounded from below by a positive constant. This finishes the proof of the lemma.

3.2. The relation between $\mathcal{A}_{m}$ and $\mathcal{A}_{-1}$. Now we will use the flow (3.1) to obtain the relation between $\mathcal{A}_{m}$ and $\mathcal{A}_{-1}$. In particular, the case $m=1$ will be served as the initial condition when we prove Theorem 1.4 by induction for odd numbers.

Proposition 3.3. Let $M$ be a closed convex $C^{2}$-hypersurface in $\mathbb{S}_{+}^{n+1}$, then the following inequality holds,

$$
\mathcal{A}_{m}(\Omega) \geq \xi_{m,-1}\left(\mathcal{A}_{-1}(\Omega)\right), \quad \forall 0 \leq m \leq n-1,
$$

where $\xi_{m,-1}$ is the unique positive function defined on $\left(0, s_{-1}\right)$ such that the equality holds if and only if $M$ is a geodesic sphere.

Proof. First of all, we can assume that the hypersurface is smooth and strictly convex, since otherwise we can use convolutions as in the proof of Corollary 1.2 in [16] to obtain a sequence of approximating smooth strictly convex hypersurfaces converging in $C^{2}$ to $M$. The inequality follows from the approximation. We will treat the equality case $\mathcal{A}_{k}(\Omega)=\xi_{k, k-2}\left(\mathcal{A}_{k-2}(\Omega)\right)$ for general $1 \leq k \leq n-1$ in the proof of Theorem 1.4.

When $k=0, F=H$ and (3.1) becomes

$$
X_{t}=\left(n \phi^{\prime}-u H\right) \nu
$$

We have the $C^{0}$ estimate (see Proposition 4.1 in [12]), $C^{1}$ estimate(see Proposition 5.2 in [12]) and the upper bound of $H$ (see Corollary 3.3 in [12]). Then we see that the convexity is preserved by Lemma 3.2. Moreover, $C^{2}$ estimate follows directly 
from the theory of quasi-linear PDE. The surfaces converge exponentially to a sphere as $t \rightarrow \infty$ in the $C^{\infty}$ topology by Theorem 1.1 in [12. Along the flow (3.1), we have

$$
\frac{d}{d t} \mathcal{A}_{-1}=\int_{M(t)}\left(n \phi^{\prime}-H u\right)=0,
$$

and

$$
\begin{aligned}
\frac{d}{d t} \mathcal{A}_{m} & =(m+1) \int_{M(t)}\left(n \phi^{\prime}-H u\right) \sigma_{m+1} d \mu \\
& \leq(m+1) \int_{M(t)}\left(n \phi^{\prime} \sigma_{m+1}-\frac{n(m+2)}{n-(m+1)} u \sigma_{m+2}\right) d \mu \\
& =0,
\end{aligned}
$$

where we have used the Newton-Maclaurin inequality (Lemma 2.2) and Minkowski identity (Proposition 2.3). Then we have

$$
\mathcal{A}_{m}(\Omega) \geq \xi_{m,-1}\left(\mathcal{A}_{-1}(\Omega)\right) \quad \text { for } \quad m=1,2, \cdots, n-1 .
$$

the equality holds only if $M$ is a geodesic sphere. By the definition of $\xi_{m,-1}$, we know that the equality holds if $M$ is a geodesic sphere. Especially,

$$
\mathcal{A}_{1}(\Omega) \geq \xi_{1,-1}\left(\mathcal{A}_{-1}(\Omega)\right),
$$

with the equality holds if and if $M$ is a geodesic sphere.

\section{Gerhardt's flow and applications}

In this section, following [4], we will use Gerhardt's flow to prove the main theorem. Gerhardt [8] considered the inverse curvature flows of strictly convex hypersurfaces in $\mathbb{S}^{n+1}$ and obtained smooth convergence of the flows to the equator.

Assume $F=\frac{\sigma_{k}}{\sigma_{k-1}}$. Then under the inverse curvature flow,

$$
X_{t}=\frac{\nu}{F}
$$

with $M(0)=\partial B_{\rho}(o)$, the geodesic spheres stay as geodesic spheres and for time $t>0, M(t)=\partial B_{\rho(t)}(o)$. We first establish a Minkowski type inequality in $\mathbb{S}^{n+1}$ without weighted factor.

Theorem 4.1. Let $M$ be a closed convex $C^{2}$-hypersurface in $\mathbb{S}_{+}^{n+1}$, then the following inequality holds,

$$
\left(\int_{M} \sigma_{1} d \mu_{g}\right)^{2} \geq \xi\left(\mathcal{A}_{0}^{2}(\Omega)\right)
$$


where $\xi$ is the unique positive function defined on $\left(0, s_{0}^{2}\right)$ such that the equality holds if and only if $M$ is a geodesic sphere. Moreover, $\xi$ satisfies that

$$
2 \frac{n-1}{n} \xi(s)=\left(2 n+2 \xi^{\prime}(s)\right) s \quad \text { for } \quad s \in\left(0, s_{0}^{2}\right) .
$$

In fact we can express $\xi$ explicitly by

$$
\xi(s)=n^{2}(n+1)^{\frac{2}{n}} \omega_{n+1}^{\frac{2}{n}} s^{\frac{n-1}{n}}-n^{2} s,
$$

where $\omega_{n+1}$ is the volume of unit ball in $\mathbb{R}^{n+1}$.

Proof. Again we assume $M$ to be smooth and strictly convex and we use the same method as we used in the proof of Proposition 3.3 .

The function $\xi$ is defined using the following relation:

$$
\left(\int_{\partial B_{\rho(t)}(o)} \sigma_{1} d \mu_{g}\right)^{2}=\xi\left(\mathcal{A}_{0}^{2}\left(B_{\rho(t)}(o)\right)\right) .
$$

Set $f=\frac{1}{H}$. Using (2.3) for $l=1$ and (2.4) for $l=0$, we have

$$
\begin{aligned}
& \frac{d}{d t}\left(\left(\int_{\partial B_{\rho(t)}(o)} \sigma_{1} d \mu_{g}\right)^{2}-\xi\left(\mathcal{A}_{0}^{2}\left(B_{\rho(t)}(o)\right)\right)\right) \\
& =2 \int_{\partial B_{\rho(t)}(o)} \sigma_{1} d \mu_{g} \int_{\partial B_{\rho(t)}(o)} \frac{1}{\sigma_{1}}\left(2 \sigma_{2}-n \sigma_{0}\right) d \mu_{g} \\
& -2 \xi^{\prime}\left(\mathcal{A}_{0}^{2}\left(B_{\rho(t)}(o)\right)\right) \mathcal{A}_{0}\left(B_{\rho(t)}(o)\right) \int_{\partial B_{\rho(t)}(o)} \sigma_{1} \frac{1}{\sigma_{1}} d \mu_{g} \\
& =2 \frac{n-1}{n}\left(\int_{\partial B_{\rho(t)}(o)} \sigma_{1} d \mu_{g}\right)^{2}-2 n \int_{\partial B_{\rho(t)}(o)} \sigma_{1} d \mu_{g} \int_{\partial B_{\rho(t)}(o)} \frac{1}{\sigma_{1}} d \mu_{g} \\
& -2 \xi^{\prime}\left(\mathcal{A}_{0}^{2}\left(B_{\rho(t)}(o)\right)\right) \mathcal{A}_{0}^{2}\left(B_{\rho(t)}(o)\right) \\
& =2 \frac{n-1}{n}\left(\int_{\partial B_{\rho(t)}(o)} \sigma_{1} d \mu_{g}\right)^{2}-2 n \mathcal{A}_{0}^{2}\left(B_{\rho(t)}(o)\right)-2 \xi^{\prime}\left(\mathcal{A}_{0}^{2}\left(B_{\rho(t)}(o)\right)\right) \mathcal{A}_{0}^{2}\left(B_{\rho(t)}(o)\right)
\end{aligned}
$$

where the last step follows from the fact that

$$
\mathcal{A}_{0}^{2}(\Omega(t)) \leq \int_{M(t)} \sigma_{1} d \mu_{g} \int_{M(t)} \frac{1}{\sigma_{1}} d \mu_{g},
$$

with the inequality strict unless $\sigma_{1}=H$ is constant on $M(t)$. Hence we obtain that the equality holds if and only if $M(t)$ is a geodesic sphere.

By (4.4), we have

$$
2 \frac{n-1}{n} \xi\left(\mathcal{A}_{0}^{2}\left(B_{\rho(t)}(o)\right)\right)-2 n \mathcal{A}_{0}^{2}\left(B_{\rho(t)}(o)\right)-2 \xi^{\prime}\left(\mathcal{A}_{0}^{2}\left(B_{\rho(t)}(o)\right)\right) \mathcal{A}_{0}^{2}\left(B_{\rho(t)}(o)\right)=0 .
$$


We can obtain that

$$
2 \frac{n-1}{n} \xi(s)=\left(2 n+2 \xi^{\prime}(s)\right) s \quad \text { for } \quad s \in\left(0, s_{0}^{2}\right) .
$$

Let $M(t)$ solve the inverse curvature flow equation $X_{t}=\frac{1}{\sigma_{1}} \nu$ with initial condition $M(0)=M$. Denote $\mathcal{A}_{k}(t)=\mathcal{A}_{k}\left(\Omega_{t}\right)$, where $\Omega_{t}$ is the domain enclosed by $M(t)$, then we have

$$
\begin{aligned}
& \frac{d}{d t}\left(\left(\int_{M(t)} \sigma_{1} d \mu_{g}\right)^{2}-\xi\left(\mathcal{A}_{0}^{2}(t)\right)\right) \\
& =2 \int_{M(t)} \sigma_{1} d \mu_{g} \int_{M(t)} \frac{1}{\sigma_{1}}\left(2 \sigma_{2}-n \sigma_{0}\right) d \mu_{g}-2 \xi^{\prime}\left(\mathcal{A}_{0}^{2}(t)\right) \mathcal{A}_{0}(t) \int_{M(t)} \sigma_{1} \frac{1}{\sigma_{1}} d \mu_{g} \\
& \leq 2 \frac{n-1}{n}\left(\int_{M(t)} \sigma_{1} d \mu_{g}\right)^{2}-2 n \int_{M(t)} \sigma_{1} d \mu_{g} \int_{M(t)} \frac{1}{\sigma_{1}} d \mu_{g}-2 \xi^{\prime}\left(\mathcal{A}_{0}^{2}(t)\right) \mathcal{A}_{0}^{2}(t) \\
& \leq 2 \frac{n-1}{n}\left(\int_{M(t)} \sigma_{1} d \mu_{g}\right)^{2}-2 n \mathcal{A}_{0}^{2}(t)-2 \xi^{\prime}\left(\mathcal{A}_{0}^{2}(t)\right) \mathcal{A}_{0}^{2}(t) \\
& =2 \frac{n-1}{n}\left(\left(\int_{M(t)} \sigma_{1} d \mu_{g}\right)^{2}-\xi\left(\mathcal{A}_{0}^{2}(t)\right)\right)
\end{aligned}
$$

We will have

$$
\frac{d}{d t}\left(e^{-2 \frac{n-1}{n} t}\left(\left(\int_{M(t)} \sigma_{1} d \mu_{g}\right)^{2}-\xi\left(\mathcal{A}_{0}^{2}(t)\right)\right) \leq 0\right.
$$

Denote

$$
Q(t)=e^{-2 \frac{n-1}{n} t}\left(\left(\int_{M(t)} \sigma_{1} d \mu_{g}\right)^{2}-\xi\left(\mathcal{A}_{0}^{2}(t)\right)\right)
$$

then

$$
\frac{d}{d t} Q(t) \leq 0
$$

Thus

$$
Q(t)-Q(0) \leq 0
$$

for all $t \in\left[0, T^{*}\right)$.

It is proved in Theorem 1.1 in ([8]) that the curvature flow converges to an equator, as $t \rightarrow T^{*}$, and with

$$
\left|\frac{\pi}{2}-\rho\right|_{m, \mathbb{S}^{n}} \leq c_{m} \Theta \quad \forall t \in\left[t_{\delta}, T^{*}\right),
$$

where $\Theta=\arccos e^{t-T^{*}}$. It follows that

$$
\begin{aligned}
\operatorname{Vol}\left(\Omega_{t}\right) & \rightarrow \operatorname{Vol}\left(B_{\frac{\pi}{2}}\right), \\
\int_{M_{t}} d \mu_{g} & \rightarrow\left|\Sigma\left(B_{\frac{\pi}{2}}\right)\right|,
\end{aligned}
$$




$$
\int_{M_{t}} \sigma_{k} d \mu_{g} \rightarrow 0 \quad \forall 1 \leq k \leq n-1
$$

as $t \rightarrow T^{*}$. By the definition of $\mathcal{A}_{k}$, we have

$$
\mathcal{A}_{k}(t) \rightarrow \mathcal{A}_{k}\left(B_{\frac{\pi}{2}}(o)\right) \quad \forall-1 \leq k \leq n-1,
$$

as $t \rightarrow T^{*}$, which implies that

$$
\lim _{t \rightarrow T^{*}} Q(t)=0
$$

Therefore, we have

$$
\left(\int_{M(0)} \sigma_{1} d \mu_{g}\right)^{2}-\xi\left(\mathcal{A}_{0}^{2}(0)\right) \geq 0
$$

i.e.,

$$
\left(\int_{M} \sigma_{1} d \mu_{g}\right)^{2}-\xi\left(\mathcal{A}_{0}^{2}(\Omega)\right) \geq 0
$$

with the equality holds only if $M$ is a geodesic sphere. By the definition of $\xi$, we know that the equality holds if $M$ is a geodesic sphere.

Finally, we will derive the explicit expression for $\xi$. First we can solve the ODE (4.2) to obtain

$$
\xi(s)=s^{\frac{n-1}{n}} \epsilon^{-\frac{n-1}{n}} \xi(\epsilon)-n^{2}\left(s-\epsilon^{\frac{1}{n}} s^{\frac{n-1}{n}}\right) .
$$

It remains to compute the $\operatorname{limit}_{\lim _{\epsilon \rightarrow 0}} \epsilon^{-\frac{n-1}{n}} \xi(\epsilon)$. For this purpose, we notice that since the metric on $\mathbb{S}^{n+1}$ is given by $d s^{2}=d \rho^{2}+\sin ^{2} \rho d z^{2}$, we see that

$$
\mathcal{A}_{0}\left(B_{\rho}(o)\right)=\left|\partial B_{\rho}(o)\right|=\sin ^{n} \rho\left|\mathbb{S}^{n}\right|=(n+1) \omega_{n+1} \sin ^{n} \rho .
$$

On the other hand, for $\partial B_{\rho}(0) \subset \mathbb{S}^{n+1}$, we have $\kappa_{1}=\kappa_{2}=\cdots \kappa_{n}=\frac{\cos \rho}{\sin \rho}$. Hence,

$$
\int_{\partial B_{\rho}(o)} \sigma_{1} d \mu_{g}=n \frac{\cos \rho}{\sin \rho}\left|\partial B_{\rho}(o)\right|=n(n+1) \omega_{n+1} \cos \rho \sin ^{n-1} \rho .
$$

Therefore, if we choose $s=\mathcal{A}_{0}^{2}\left(B_{\rho}(o)\right)=(n+1)^{2} \omega_{n+1}^{2} \sin ^{2 n} \rho$, then

$$
\begin{aligned}
\xi(s) & =\left(\int_{\partial B_{\rho}(o)} \sigma_{1} d \mu_{g}\right)^{2}=n^{2}(n+1)^{2} \omega_{n+1}^{2} \cos ^{2} \rho \sin ^{2(n-1)} \rho \\
& =n^{2}(n+1)^{2} \omega_{n+1}^{2} \cos ^{2} \rho\left(\frac{s}{(n+1)^{2} \omega_{n+1}^{2}}\right)^{\frac{n-1}{n}}=n^{2}(n+1)^{\frac{2}{n}} \omega_{n+1}^{\frac{2}{n}} \cos ^{2} \rho s^{\frac{n-1}{n}} .
\end{aligned}
$$

Since $\rho \rightarrow 0$ as $s \rightarrow 0$, we see that

$$
\lim _{\epsilon \rightarrow 0} \epsilon^{-\frac{n-1}{n}} \xi(\epsilon)=\lim _{\rho \rightarrow 0}\left(n^{2}(n+1)^{\frac{2}{n}} \omega_{n+1}^{\frac{2}{n}} \cos ^{2} \rho\right)=n^{2}(n+1)^{\frac{2}{n}} \omega_{n+1}^{\frac{2}{n}} .
$$

Now (4.3) follows by letting $\epsilon \rightarrow 0$ in (4.6).

Now we will establish the relation between $\mathcal{A}_{2}$ and $\mathcal{A}_{0}$. 
Proposition 4.2. Let $M$ be a closed convex $C^{2}$-hypersurface in $\mathbb{S}_{+}^{n+1}$, then the following inequality holds,

$$
\mathcal{A}_{2}(\Omega) \geq \xi_{2,0}\left(\mathcal{A}_{0}(\Omega)\right)
$$

where $\xi_{2,0}$ is the unique positive function defined on $\left(0, s_{0}\right)$ such that the equality holds if and only if $M$ is a geodesic sphere. Moreover, $\xi_{2,0}$ satisfies that

$$
\xi_{2,0}^{\prime}(s)=\frac{(n-2) \xi_{2,0}(s)-(n-1) s}{n s} .
$$

In fact we can express $\xi_{2,0}$ explicitly by

$$
\xi_{2,0}(s)=\frac{n(n-1)}{2}(n+1)^{\frac{2}{n}} \omega_{n+1}^{\frac{2}{n}} s^{\frac{n-2}{n}}-\frac{n-1}{2} s,
$$

where $\omega_{n+1}$ is the volume of unit ball in $\mathbb{R}^{n+1}$.

Proof. Again we assume $M$ to be smooth and strictly convex and we use the same method as we used in the proof of Proposition 3.3.

For $0<\rho(t)<\frac{\pi}{2}$, the function $\xi_{2,0}$ is defined by

$$
\mathcal{A}_{2}\left(B_{\rho(t)}(o)\right)-\xi_{2,0}\left(\mathcal{A}_{0}\left(B_{\rho(t)}(o)\right)\right)=0 .
$$

Using (2.4) for $l=0$ and $l=2$ and considering the flow (4.1) with $F=\frac{\sigma_{2}}{\sigma_{1}}$, we have

$$
\begin{aligned}
& \frac{d}{d t}\left(\mathcal{A}_{2}\left(B_{\rho(t)}(o)\right)-\xi_{2,0}\left(\mathcal{A}_{0}\left(B_{\rho(t)}(o)\right)\right)\right. \\
& =3 \int_{\partial B_{\rho(t)}(o)} \sigma_{3} \frac{1}{\frac{\sigma_{2}}{\sigma_{1}}} d \mu_{g}-\xi_{2,0}^{\prime}\left(\mathcal{A}_{0}\left(B_{\rho(t)}(o)\right)\right) \int_{\partial B_{\rho(t)}(o)} \sigma_{1} \frac{1}{\frac{\sigma_{2}}{\sigma_{1}}} d \mu_{g} \\
& =\frac{2(n-2)}{n-1} \int_{\partial B_{\rho(t)}(o)} \sigma_{2} d \mu_{g}-\frac{2 n}{n-1} \xi_{2,0}^{\prime}\left(\mathcal{A}_{0}\left(B_{\rho(t)}(o)\right)\right) \int_{\partial B_{\rho(t)}(o)} \sigma_{0} d \mu_{g},
\end{aligned}
$$

where the last step follows from the fact that the geodesic sphere is totally umbilic. (4.11) yields that

$$
\frac{2(n-2)}{n-1} \int_{\partial B_{\rho(t)}(o)} \sigma_{2} d \mu_{g}=\frac{2 n}{n-1} \xi_{2,0}^{\prime}\left(\mathcal{A}_{0}\left(B_{\rho(t)}(o)\right)\right) \int_{\partial B_{\rho(t)}(o)} \sigma_{0} d \mu_{g} .
$$

From the definition of $\mathcal{A}_{k}$, we have

$$
\mathcal{A}_{2}\left(B_{\rho(t)}(o)\right)=\int_{\partial B_{\rho(t)}(o)} \sigma_{2} d \mu_{g}+\frac{K(n-1)}{2-1} \mathcal{A}_{0}\left(B_{\rho(t)}(o)\right) .
$$

Combining (4.11), (4.12) and (4.13), we have

$$
\xi_{2,0}^{\prime}(s)=\frac{(n-2)\left(\xi_{2,0}(s)-(n-1) s\right)}{n s} .
$$


Let $M(t)$ solve the inverse curvature flow equation $X_{t}=\frac{\sigma_{1}}{\sigma_{2}} \nu$ with initial condition $M(0)=M$. Denote $\mathcal{A}_{k}(t)=\mathcal{A}_{k}\left(\Omega_{t}\right)$, where $\Omega_{t}$ is the domain enclosed by $M(t)$, then we have

$$
\begin{aligned}
& \frac{d}{d t}\left(\mathcal{A}_{2}(t)-\xi_{2,0}\left(\mathcal{A}_{0}(t)\right)\right) \\
& =3 \int_{M(t)} \sigma_{3} \frac{1}{\frac{\sigma_{2}}{\sigma_{1}}} d \mu_{g}-\xi_{2,0}^{\prime}\left(\mathcal{A}_{0}(t)\right) \int_{M(t)} \sigma_{1} \frac{1}{\frac{\sigma_{2}}{\sigma_{1}}} d \mu_{g} \\
& \leq \frac{2(n-2)}{n-1} \int_{M(t)} \sigma_{2} d \mu_{g}-\frac{2 n}{n-1} \xi_{2,0}^{\prime}\left(\mathcal{A}_{0}(t)\right) \int_{M(t)} \sigma_{0} d \mu_{g} \\
& =\frac{2(n-2)}{n-1}\left(\mathcal{A}_{2}(t)-\frac{K(n-1)}{2-1} \mathcal{A}_{0}(t)\right)-\frac{2 n}{n-1} \xi_{2,0}^{\prime}\left(\mathcal{A}_{0}(t)\right) \mathcal{A}_{0}(t) \\
& =\frac{2(n-2)}{n-1}\left(\mathcal{A}_{2}(t)-\frac{K(n-1)}{2-1} \mathcal{A}_{0}(t)\right) \\
& -\frac{2 n}{n-1} \frac{(n-2) \xi_{2,0}\left(\mathcal{A}_{0}(t)\right)-(n-1) \mathcal{A}_{0}(t)}{n \mathcal{A}_{0}(t)} \mathcal{A}_{0}(t) \\
& =\frac{2(n-2)}{n-1}\left(\mathcal{A}_{2}(t)-\xi_{2,0}\left(\mathcal{A}_{0}(t)\right)\right) .
\end{aligned}
$$

We have

$$
\frac{d}{d t}\left(e^{-\frac{2(n-2)}{n-1} t}\left(\mathcal{A}_{2}(t)-\xi_{2,0}\left(\mathcal{A}_{0}(t)\right)\right)\right) \leq 0
$$

Denote

$$
Q_{2}(t)=e^{-\frac{2(n-2)}{n-1} t}\left(\mathcal{A}_{2}(t)-\xi_{2,0}\left(\mathcal{A}_{0}(t)\right)\right)
$$

then

$$
\frac{d}{d t} Q_{2}(t) \leq 0
$$

Thus

$$
Q_{2}(t)-Q_{2}(0) \leq 0
$$

for all $t \in\left[0, T^{*}\right)$.

By the definition of $\mathcal{A}_{k}$, we have

$$
\lim _{t \rightarrow T^{*}} Q(t)=0
$$

Therefore, we have

$$
\mathcal{A}_{2}(0)-\xi_{2,0}\left(\mathcal{A}_{0}(0)\right) \geq 0
$$

i.e.,

$$
\mathcal{A}_{2}(\Omega) \geq \xi_{2,0}\left(\mathcal{A}_{0}(\Omega)\right),
$$

the equality holds only if $M$ is a geodesic sphere. By the definition of $\xi_{2,0}$, we know that the equality holds if $M$ is a geodesic sphere. 
Finally, we will derive the explicit expression for $\xi_{2,0}$. First we can solve the ODE (4.9) to obtain

$$
\xi_{2,0}(s)=s^{\frac{n-2}{n}} \epsilon^{-\frac{n-2}{n}} \xi_{2,0}(\epsilon)-\frac{n-1}{2}\left(s-\epsilon^{\frac{2}{n}} s^{\frac{n-2}{n}}\right) .
$$

It remains to compute the $\operatorname{limit}_{\lim _{\epsilon \rightarrow 0}} \epsilon^{-\frac{n-2}{n}} \xi_{2,0}(\epsilon)$. For this purpose, recall that the area of the geodesic sphere is given by (4.7). On the other hand, for $\partial B_{\rho}(0) \subset$ $\mathbb{S}^{n+1}$, we have $\kappa_{1}=\kappa_{2}=\cdots \kappa_{n}=\frac{\cos \rho}{\sin \rho}$. Hence, we have $\sigma_{2}=\frac{n(n-1)}{2} \frac{\cos ^{2} \rho}{\sin ^{2} \rho}$ so that

$$
\begin{aligned}
\mathcal{A}_{2}\left(B_{\rho}(o)\right) & =\int_{\partial B_{\rho}(o)} \sigma_{2} d \mu_{g}+(n-1) \mathcal{A}_{0}\left(B_{\rho}(o)\right) \\
& =\frac{n(n-1)}{2} \frac{\cos ^{2} \rho}{\sin ^{2} \rho}\left|\partial B_{\rho}(o)\right|+(n-1)\left|\partial B_{\rho}(o)\right| .
\end{aligned}
$$

Therefore, if we choose $s=\mathcal{A}_{0}\left(B_{\rho}(o)\right)=\left|\partial B_{\rho}(o)\right|=(n+1) \omega_{n+1} \sin ^{n} \rho$, then

$$
\begin{aligned}
\xi_{2,0}(s) & =\mathcal{A}_{2}\left(B_{\rho}(o)\right)=\frac{n(n-1)}{2} \frac{\cos ^{2} \rho}{\sin ^{2} \rho} s+(n-1) s \\
& =\frac{n(n-1)}{2} \cos ^{2} \rho s\left(\frac{s}{(n+1) \omega_{n+1}}\right)^{-\frac{2}{n}}+(n-1) s \\
& =\frac{n(n-1)}{2}(n+1)^{\frac{2}{n}} \omega_{n+1}^{\frac{2}{n}} \cos ^{2} \rho s^{\frac{n-2}{n}}+(n-1) s .
\end{aligned}
$$

Since $\rho \rightarrow 0$ as $s \rightarrow 0$, we see that

$$
\begin{aligned}
\lim _{\epsilon \rightarrow 0} \epsilon^{-\frac{n-2}{n}} \xi_{2,0}(\epsilon) & =\lim _{\rho \rightarrow 0}\left(\frac{n(n-1)}{2}(n+1)^{\frac{2}{n}} \omega_{n+1}^{\frac{2}{n}} \cos ^{2} \rho+(n-1) \epsilon^{\frac{2}{n}}\right) \\
& =\frac{n(n-1)}{2}(n+1)^{\frac{2}{n}} \omega_{n+1}^{\frac{2}{n}} .
\end{aligned}
$$

Now (4.10) follows by letting $\epsilon \rightarrow 0$ in (4.14).

Remark 4.1. We can compare Theorem 4.1 and Proposition 4.2 with Theorem 1.5 (see (1.5) and (1.6)) of [16]. The method we used to deduce Theorem 4.1 and Proposition 4.2 can be used to derive the relation between $\mathcal{A}_{k}$ and $\mathcal{A}_{k-2}$ for general $1 \leq k \leq n-1$.

Next, we will study the properties of the function $\xi_{k, k-2}$, which will be used in the proof of the main theorem.

Proposition 4.3. For any $s \in\left(0, s_{k-2}\right)$, the following holds

$$
\xi_{k, k-2}^{\prime}(s)=\frac{n-k}{n-k+2} \frac{\xi_{k, k-2}(s)-K \frac{n-k+1}{k-1} s}{s-K \frac{n-k+3}{k-3} \xi_{k-2, k-4}^{-1}(s)} \quad \text { for } \quad k \geq 3,
$$

where $\xi_{k, k-2}$ and $\xi_{k-2, k-4}$ are defined as in (2.5). 
Proof. For $k \geq 3$ and $0<\rho(t)<\frac{\pi}{2}$, by the definition of $\xi_{k, k-2}$, we have

$$
\mathcal{A}_{k}\left(B_{\rho(t)}(o)\right)-\xi_{k, k-2}\left(\mathcal{A}_{k-2}\left(B_{\rho(t)}(o)\right)\right)=0 .
$$

By (2.4), we have along the flow (4.1) with $F=\frac{\sigma_{k}}{\sigma_{k-1}}$

$$
\begin{aligned}
& \frac{d}{d t}\left(\mathcal{A}_{k}\left(B_{\rho(t)}(o)\right)-\xi_{k, k-2}\left(\mathcal{A}_{k-2}\left(B_{\rho(t)}(o)\right)\right)\right. \\
& =(k+1) \int_{\partial B_{\rho(t)}(o)} \sigma_{k+1} \frac{1}{\frac{\sigma_{k}}{\sigma_{k-1}}} d \mu_{g} \\
& -(k-1) \xi_{k, k-2}^{\prime}\left(\mathcal{A}_{k-2}\left(B_{\rho(t)}(o)\right)\right) \int_{\partial B_{\rho(t)}(o)} \sigma_{k-1} \frac{1}{\frac{\sigma_{k}}{\sigma_{k-1}}} d \mu_{g} \\
& =\frac{k(n-k)}{n-k+1} \int_{\partial B_{\rho(t)}(o)} \sigma_{k} d \mu_{g} \\
& -\frac{(n-k+2) k}{n-k+1} \xi_{k, k-2}^{\prime}\left(\mathcal{A}_{k-2}\left(B_{\rho(t)}(o)\right)\right) \int_{\partial B_{\rho(t)}(o)} \sigma_{k-2} d \mu_{g},
\end{aligned}
$$

where the last step follows from the fact that the geodesic sphere is totally umbilic. (4.16) yields that

$$
\frac{k(n-k)}{n-k+1} \int_{\partial B_{\rho(t)}(o)} \sigma_{k} d \mu_{g}=\frac{(n-k+2) k}{n-k+1} \xi_{k, k-2}^{\prime}\left(\mathcal{A}_{k-2}\left(B_{\rho(t)}(o)\right)\right) \int_{\partial B_{\rho(t)}(o)} \sigma_{k-2} d \mu_{g} .
$$

From the definition of $\mathcal{A}_{k}$, we have

$$
\begin{aligned}
\mathcal{A}_{k}\left(B_{\rho(t)}(o)\right) & =\int_{\partial B_{\rho(t)}(o)} \sigma_{k} d \mu_{g}+K \frac{n-k+1}{k-1} \mathcal{A}_{k-2}\left(B_{\rho(t)}(o)\right) \\
\mathcal{A}_{k-2}\left(B_{\rho(t)}(o)\right) & =\int_{\partial B_{\rho(t)}(o)} \sigma_{k-2} d \mu_{g}+K \frac{n-k+3}{k-3} \mathcal{A}_{k-4}\left(B_{\rho(t)}(o)\right)
\end{aligned}
$$

Under the assumption that

$$
\mathcal{A}_{k-2}\left(B_{\rho}(o)\right)=\xi_{k-2, k-4}\left(\mathcal{A}_{k-4}\left(B_{\rho}(o)\right)\right),
$$

we have

$$
\begin{aligned}
& \frac{k(n-k)}{n-k+1}\left(\xi_{k, k-2}\left(\mathcal{A}_{k-2}\left(B_{\rho(t)}(o)\right)\right)-K \frac{n-k+1}{k-1} \mathcal{A}_{k-2}\left(B_{\rho(t)}(o)\right)\right) \\
& -\frac{(n-k+2) k}{n-k+1}\left(\mathcal{A}_{k-2}\left(B_{\rho(t)}(o)\right)-\frac{K(n-k+3)}{k-3} \xi_{k-2, k-4}^{-1}\left(\mathcal{A}_{k-2}\left(B_{\rho(t)}(o)\right)\right)\right) . \\
& \xi_{k, k-2}^{\prime}\left(\mathcal{A}_{k-2}\left(B_{\rho(t)}(o)\right)\right)=0 .
\end{aligned}
$$

Then we have

$$
\xi_{k, k-2}^{\prime}(s)=\frac{n-k}{n-k+2} \frac{\xi_{k, k-2}(s)-K \frac{n-k+1}{k-1} s}{s-K \frac{n-k+3}{k-3} \xi_{k-2, k-4}^{-1}(s)}
$$


for any $s \in\left(0, s_{k-2}\right)$.

Now we can prove our main result.

Proof of Theorem 1.4. Case 1. $M$ is a closed strictly convex and smooth hypersurface. We will prove Theorem 1.4 by reduction.

Let $M(t)$ solve the inverse curvature flow equation $X_{t}=\frac{\sigma_{k-1}}{\sigma_{k}} \nu$ with initial condition $M(0)=M$. Denote $\mathcal{A}_{k}(t)=\mathcal{A}_{k}\left(\Omega_{t}\right)$, where $\Omega_{t}$ is the domain enclosed by $M(t)$. For $k=1,2$, the inequality (1.3) holds by Proposition 3.3 with $m=1$ and Proposition 4.2, respectively. Then we can assume that

$$
\mathcal{A}_{k-2}(\Omega) \geq \xi_{k-2, k-4}\left(\mathcal{A}_{k-4}(\Omega)\right),
$$

for any strictly convex hypersurface $M$ in $\mathbb{S}^{n+1}$ and the equality holds if and only if $M$ is a geodesic sphere. Since

$$
\frac{d}{d t}\left(\mathcal{A}_{k-2}(t)\right)=(k-1) \int_{M(t)} \sigma_{k-1} \frac{1}{\frac{\sigma_{k}}{\sigma_{k-1}}} d \mu_{g} \geq \frac{k(n-k+2)}{n-k+1} \int_{M(t)} \sigma_{k-2} d \mu_{g}>0
$$

and $M(t)$ converges to an equator of $\mathbb{S}^{n+1}$, we have

$$
\mathcal{A}_{k-2}(t) \in\left(0, s_{k}\right) \text {. }
$$

By Proposition 4.3 and applying Newton-Maclaurin inequality, we have

$$
\begin{aligned}
& \frac{d}{d t}\left(\mathcal{A}_{k}(t)-\xi_{k, k-2}\left(\mathcal{A}_{k-2}(t)\right)\right) \\
= & (k+1) \int_{M(t)} \sigma_{k+1} \frac{1}{\frac{\sigma_{k}}{\sigma_{k-1}}} d \mu_{g}-(k-1) \xi_{k, k-2}^{\prime}\left(\mathcal{A}_{k-2}(t)\right) \int_{M(t)} \sigma_{k-1} \frac{1}{\frac{\sigma_{k}}{\sigma_{k-1}}} d \mu_{g} \\
\leq & \frac{k(n-k)}{n-k+1} \int_{M(t)} \sigma_{k} d \mu_{g}-\frac{(n-k+2) k}{n-k+1} \xi_{k, k-2}^{\prime}\left(\mathcal{A}_{k-2}(t)\right) \int_{M(t)} \sigma_{k-2} d \mu_{g} \\
= & \frac{k(n-k)}{n-k+1}\left(\mathcal{A}_{k}(t)-K \frac{n-k+1}{k-1} \mathcal{A}_{k-2}(t)\right) \\
& -\frac{(n-k+2) k}{n-k+1} \xi_{k, k-2}^{\prime}\left(\mathcal{A}_{k-2}(t)\right)\left(\mathcal{A}_{k-2}(t)-K \frac{n-k+3}{k-3} \mathcal{A}_{k-4}(t)\right) \\
= & \frac{k(n-k)}{n-k+1}\left(\mathcal{A}_{k}(t)-K \frac{n-k+1}{k-1} \mathcal{A}_{k-2}(t)\right) \\
& -\frac{(n-k+2) k}{n-k+1} \frac{n-k}{n-k+2} \frac{\xi_{k, k-2}\left(\mathcal{A}_{k-2}(t)\right)-K \frac{n-k+1}{k-1} \mathcal{A}_{k-2}(t)}{\mathcal{A}_{k-2}(t)-K \frac{n-k+3}{k-3} \xi_{k-2, k-4}^{-1}\left(\mathcal{A}_{k-2}(t)\right)} \\
& \left(\mathcal{A}_{k-2}(t)-K \frac{n-k+3}{k-3} \mathcal{A}_{k-4}(t)\right) \\
\leq & \frac{k(n-k)}{n-k+1}\left(\mathcal{A}_{k}(t)-K \frac{n-k+1}{k-1} \mathcal{A}_{k-2}(t)\right) \\
& -\frac{k(n-k)}{n-k+1}\left(\xi_{k, k-2}\left(\mathcal{A}_{k-2}(t)\right)-K \frac{n-k+1}{k-1} \mathcal{A}_{k-2}(t)\right)
\end{aligned}
$$


where in the last step we use the assumption that

$$
\mathcal{A}_{k-4}(t) \leq \xi_{k-2, k-4}^{-1}\left(\mathcal{A}_{k-2}(t)\right)
$$

Therefore we have

$$
\frac{d}{d t}\left(\mathcal{A}_{k}(t)-\xi_{k, k-2}\left(\mathcal{A}_{k-2}(t)\right) \leq \frac{k(n-k)}{n-k+1}\left(\mathcal{A}_{k}(t)-\xi_{k, k-2}\left(\mathcal{A}_{k-2}(t)\right)\right) .\right.
$$

Assume that

$$
Q_{k}(t)=e^{-\frac{k(n-k)}{n-k+1} t}\left(\mathcal{A}_{k}(t)-\xi_{k, k-2}\left(\mathcal{A}_{k-2}(t)\right)\right)
$$

then

$$
\frac{d}{d t} Q_{k}(t) \leq 0
$$

Thus

$$
Q_{k}(t)-Q_{k}(0) \leq 0
$$

for all $t \in\left[0, T^{*}\right)$.

By the definition of $\mathcal{A}_{k}$, we have

$$
\lim _{t \rightarrow T^{*}} Q_{k}(t)=0
$$

Therefore,

$$
\mathcal{A}_{k}(0)-\xi_{k, k-2}\left(\mathcal{A}_{k-2}(0)\right) \geq 0
$$

i.e.,

$$
\mathcal{A}_{k}(\Omega) \geq \xi_{k, k-2}\left(\mathcal{A}_{k-2}(\Omega)\right)
$$

the equality holds only if $M$ is a geodesic sphere. By the definition of $\xi_{k, k-2}$, we know that the equality holds if $M$ is a geodesic sphere.

Case 2. $M$ is a closed convex $C^{2}$-hypersurface.

We can obtain a sequence of approximating smooth strictly convex hypersurfaces converging in $C^{2}$ to $M$. The inequality follows from the approximation. We now treat the equality case $\mathcal{A}_{k}(\Omega)=\xi_{k, k-2}\left(\mathcal{A}_{k-2}(\Omega)\right)$ for general $1 \leq k \leq n-1$.

Assume $M$ is convex and the equality holds for general $1 \leq k \leq n-1$, we will show $M$ is strictly $k$-convex. To see this, note that both $\mathcal{A}_{k}$ and $\mathcal{A}_{k-2}$ are positive, since there exists at least one elliptic point on a closed hypersurface in $\mathbb{S}_{+}^{n+1}$. Let $M_{+}=\left\{x \in M \mid \sigma_{k}(x)>0\right\} . M_{+}$is open and nonempty. We claim that $M_{+}$is closed. This would imply $M=M_{+}$, so $M$ is strictly $k$ convex.

We now prove that $M_{+}$is closed. We will follow the idea of [11]. Pick any $\eta \in C_{0}^{2}\left(M_{+}\right)$compactly supported in $M_{+}$. Let $M_{s}$ be the hypersurface determined by position function $X_{s}=X+s \eta \nu$, where $X$ is the position function of $M$ and $\nu$ is the unit outernormal of $M$ at $X$. Let $\Omega_{s}$ be the domain enclosed by $M_{s}$. It is easy to show that $M_{s}$ is $k$-convex when $s$ is small enough. Define

$$
\mathcal{I}_{k}\left(\Omega_{s}\right)=\mathcal{A}_{k}\left(\Omega_{s}\right)-\xi_{k, k-2}\left(\mathcal{A}_{k-2}\left(\Omega_{s}\right)\right) \text {. }
$$

Therefore $\mathcal{I}_{k}\left(\Omega_{s}\right)-\mathcal{I}_{k}(\Omega) \geq 0$ for $s$ small, which implies that

$$
\left.\frac{d}{d s} \mathcal{I}_{k}\left(\Omega_{s}\right)\right|_{s=0}=0 \text {. }
$$


Simple calculation yields

$$
\partial_{t} \mathcal{A}_{l}=(l+1) \int_{M} f \sigma_{l+1} .
$$

Therefore,

$$
\left.\frac{d}{d s} \mathcal{I}_{k}\left(\Omega_{s}\right)\right|_{s=0}=(k+1) \int_{M}\left(\sigma_{k+1}-c_{1} \sigma_{k-1}\right) \eta d \mu_{g}=0,
$$

where $c_{1}=\frac{k-1}{k+1} \xi_{k, k-2}^{\prime}\left(\mathcal{A}_{k-2}\right)>0$ and for any $\eta \in C_{0}^{2}\left(M_{+}\right)$. Thus,

$$
\sigma_{k+1}=c_{1} \sigma_{k-1}, \quad \forall x \in M_{+} .
$$

It follows from the Newton-Maclaurine inequality that there is a dimensional constant $C(n, k)$ such that

$$
\sigma_{k+1} \leq C(n, k) \sigma_{k-1}^{1+\frac{2}{k-1}}(x), \quad x \in M_{+} .
$$

By (4.17), there is a positive $c_{2}$, such that

$$
\sigma_{k-1} \geq c_{2}>0, \quad \forall x \in M_{+},
$$

where $c_{2}=\left(\frac{c_{1}}{C(n, k)}\right)^{\frac{k-1}{2}}$ is a positive constant depending only on $n, k$ and $\Omega$. In view of (4.17), we have

$$
\sigma_{k+1} \geq c_{1} c_{2}, \quad \forall x \in M_{+},
$$

which implies that

$$
\sigma_{k} \geq c_{3}>0
$$

where $c_{3}=\sqrt{\frac{(n-k+1)(k+1)}{k(n-k)} c_{1} c_{2}^{2}}$ is a positive constant depending only on $n, k$ and $\Omega$.. It follows that $M_{+}$is closed.

Then we claim that the flow (3.1) preserves the convexity in a short time. We denote $M$ by $M_{0}$. Approximate the initial surface $M_{0}$ by a strictly convex ones $M_{0}^{\epsilon}$, by the implicit function theorem, there exists a $t_{0}>0$ (independent of $\epsilon$ ) such that (3.1) has a regular solution $M^{\epsilon}(t)$ (with $C^{0}, C^{1}, C^{2}$ bounds) which satisfies that $M^{\epsilon}(0)=M_{0}^{\epsilon}$ for $0 \leq t \leq t_{0}$. Strict convexity is preserved for the approximate flows $M^{\epsilon}(t)$ by Lemma 3.2. Letting $\epsilon \rightarrow 0$, we could obtain that $M(t)$ is convex for any $0 \leq t \leq t_{0}$.

Now we will prove that the equality $\mathcal{A}_{k}\left(\Omega_{t}\right)=\xi_{k, k-2}\left(\mathcal{A}_{k-2}\left(\Omega_{t}\right)\right)$ would be preserved at least in a short time along the flow (3.1). On one hand, by (2.4), we could obtain that

$$
\frac{d}{d t} \mathcal{A}_{k}=(k+1) \int\left(c_{n, k} \phi^{\prime} \sigma_{k+1}-\frac{\sigma_{k+1}^{2}}{\sigma_{k}}\right) \leq 0,
$$

and

$$
\frac{d}{d t} \mathcal{A}_{k-2}=(k-1) \int\left(c_{n, k} \phi^{\prime} \sigma_{k-1}-\frac{\sigma_{k+1} \sigma_{k-1}}{\sigma_{k}}\right) \geq 0 .
$$


It implies that $\mathcal{A}_{k}\left(\Omega_{t}\right) \leq \mathcal{A}_{k}(\Omega)$ and $\mathcal{A}_{k-2}(\Omega) \leq \mathcal{A}_{k-2}\left(\Omega_{t}\right)$ for any $0 \leq t \leq t_{0}$. Since $\mathcal{A}_{k}(\Omega)=\xi_{k, k-2}\left(\mathcal{A}_{k-2}(\Omega)\right)$, we have

$$
\mathcal{A}_{k}\left(\Omega_{t}\right) \leq \mathcal{A}_{k}(\Omega)=\xi_{k, k-2}\left(\mathcal{A}_{k-2}(\Omega)\right) \leq \xi_{k, k-2}\left(\mathcal{A}_{k-2}\left(\Omega_{t}\right)\right)
$$

along the flow (3.1). On the other hand, since the convexity is preserved, we could obtain the inequality $\mathcal{A}_{k}\left(\Omega_{t}\right) \geq \xi_{k, k-2}\left(\mathcal{A}_{k-2}\left(\Omega_{t}\right)\right)$.

As a result, the equality of the Newton-Maclaurin inequality must be held at every point of $\mathrm{M}(\mathrm{t})$ for each $0 \leq t \leq t_{0}$. This implies that $M(t)$ is a geodesic sphere for each $0 \leq t \leq t_{0}$. In particular, $M_{0}$ is a geodesic sphere.

This finishes the proof of the theorem.

Acknowledgements. The authors would like to thank Professor Jiayu Li and Professor Pengfei Guan for helpful suggestions. The authors would also be grateful for useful discussions with Professor Yong Wei.

\section{REFERENCES}

[1] A.D. Alexandrov, Zur Theorie der gemischten Volumina von konexen Körpern, II. Neue Ungleichungen zwischen den gemischten Volumina und ihre Anwendungen, Mat. Sb.(N.S.), 2 (1937), 1205-1238 (in Russian).

[2] A.D. Alexandrov, Zur Theorie der gemischten Volumina von konexen Körpern, III. Die Erweiterung zweeier Lehrsatze Minkowski über die konvexen Polyeder auf beliebige konvexe Flachen, Mat. Sb.(N.S.), 3 (1938), 27-46 (in Russian).

[3] B. Andrews, X. Chen, Y. Wei, Volume preserving flow and Alexandrov-Fenchel type inequalities in hyperbolic space, J. Euro. Math. Soc., arXiv: 1805.11776v1 (to appear).

[4] S. Brendle, P. Guan and J. Li, An inverse curvature type hypersurface flow in space forms, preprint.

[5] S. Brendle, P. Hung and M. Wang, A Minkowski inequality for hypersurfaces in the anti-de Sitter-Schwarzschild manifold, Comm. Pure Appl. Math., 69 (2016), no. 1, 124-144.

[6] C. Chen, P. Guan, J. Li and J. Scheuer, A curvature hypersurface flow in $\mathbb{S}^{n+1}$, work in progress.

[7] Y. Ge, G. Wang and J. Wu, Hyperbolic Alexandrov-Fenchel quermassintegral inequalities, II, J. Diff. Geom., 98 (2014), no. 2, 69-96.

[8] C. Gerhardt, Curvature flows in the sphere, J. Differ. Geom., 100 (2014), 301-347.

[9] F. Girão and N. Pinheiro, An Alexandrov-Fenchel type inequality for hypersurfaces in the sphere, Ann. Glob.Anal.Geom., 52 (2017), 413-424.

[10] P. Guan, Curvature measures, isoperimetric inequalities and fully nonlinear PDEs, "Fully Nonlinear PDEs in Real and Complex Geometry and Optics", Cetraro, Italy 2012, Editors: Cristian E.Gutiarrez, Ermannno Lanconelli, Springer(2014), 47-94.

[11] P. Guan and J. Li, The quermassintegral inequalities for $k$-convex starshaped domains, Adv. Math., 221 (2009), no. 5, 1725-1732.

[12] P. Guan and J. Li, A mean curvature type flow in space forms, Int. Math. Res. Not., (2015), no. $13,4716-4740$. 
[13] P. Guan and J. Li, Isoperimetric type inequalities and hypersurface flows, J. Math. Study. A special issue on the occasion of 70th birthdays of professors A. Chang and P. Yang. http:// www. math. mcgill.ca/guan/Guan-Li-2019S1.pdf.

[14] Y. Hu, H. Li and Y. Wei, Locally constraint inverse curvature flows in hyperbolic space, Math. Annal., arXiv: 2002.10643v2 (to appear).

[15] H. Li, Y. Wei and C. Xiong, A geometric inequality on hypersurface in hyperbolic space, Adv. Math., 253 (2014), 152-162.

[16] M. Makowski and J. Scheuer, Rigidity results, inverse curvature flows and AlexandrovFenchel type inequalities in the sphere, Asian J. Math., 20 (2016), no. 5, 869-892.

[17] J. Parbosa and A. Colares, Stability of hypersurfaces with constant r-mean curvature, Ann. Global Anal. Geom., 15 (1997), no. 3, 277-297.

[18] G. Solanes, Integral geometry and the Gauss-Bonnet theorem in constant curvature spaces, Trans. Amer. Math. Soc., 358 (2006), no. 3, 1105-1115.

[19] J. Urbas, An expansion of convex hypersurfaces, J.Differ. Geom., 33 (1991), no. 1, 91-125.

[20] G. Wang and C. Xia, Isoperimetric type problems and Alexandrov-Fenchel type inequalities in the hyperbolic space, Adv. Math., 259 (2014), 523-556.

[21] Y. Wei and C. Xiong, Alexandrov-Fenchel type inequalities for convex hypersurfaces in hyperbolic space and in sphere, Pac. J. Math., 277 (2015), no. 1, 219-239.

Min Chen, University of Science and Technology of China, No.96, JinZhai Road Baohe District,Hefei, Anhui, 230026,P.R.China.

Email address: cmcm@mail.ustc.edu.cn

Jun Sun, School of Mathematics and Statistics, Wuhan University, Wuhan, and Hubei Key Laboratory of Computational Science (Wuhan University), Wuhan, 430072, P. R. OF China.

Email address: sunjun@whu.edu.cn 